\title{
Fault Detection in Transmission Lines - A Novel Voltage-Based Scheme for Differential Protection
}

\author{
Safa Kareem Al-Sachit \\ Electrical and Computer Engineering, \\ University of Auckland \\ Auckland, New Zealand \\ slas931@aucklanduni.ac.nz
}

\author{
Mohammad Javad Sanjari \\ Electrical and Computer Engineering, \\ University of Auckland \\ Auckland, New Zealand \\ msan310@aucklanduni.ac.nz
}

\author{
Nirmal-Kumar C Nair \\ Electrical and Computer Engineering, \\ University of Auckland \\ Auckland, New Zealand \\ n.nair@auckland.ac.nz
}

\begin{abstract}
Current-based protection schemes such as distance, overcurrent, and differential relays are usually used to protect transmission lines (TL) in power systems where the high fault current plays a key role in detecting faults. The continuous development in the power network and emerging new technologies have made the power grid more complicated and soon will start to affect the reliability of the existing protection schemes. Issues like current transformer saturation, the effect of the mutual coupling impedance of the TL and emerging new power electronic-based technologies have become major challenges in power systems from a protection perspective. To avoid all the current-based problems this paper proposes a new voltage-based relay principle for TL protection to indicate fault occurrences in transmission networks. The proposed scheme is tested under all fault events to show that it is highly accurate when it comes to rapid trip activation during any of the tested cases.
\end{abstract}

Keywords: Differential relay, negative sequence voltage, relay modeling, symmetrical components, transmission line protection, unbalanced fault.

\section{INTRODUCTION}

$\mathrm{T}$ ransmission lines are subject to many events that might cause small or bulk damage to them and to the other parts of the system. Events which might affect TL include current transformer (CT) saturation issue, zero current mutual coupling, fault current limitations of power electronic based devices (PED), and grid code obligations. As a result, a sensitive, reliable and fast protection scheme is required to reduce expected damage. Many protection strategies have been suggested for TL in high, medium and low voltage parts of the network. These protection schemes such as overcurrent relay (OCR), distance relay and differential relay, are designed based on using current generally as an indicator for fault detecting and fault locating in power systems.

OCR simply detects faults when the current in TL exceeds the predetermined setting value. Reference [1] shows the OCR selectivity and ability to avoid unwanted tripping in a radial unidirectional network; however, it still cannot deal with meshed distribution networks containing distributed generators due to the bidirectional current even when using a directional element. Since OCR is a current-based protection scheme, it also suffers from the issue of low level of fault current contributed to the system by PEDs, which might affect OCR scheme discrimination. Reference [2] also emphasizes that OCR in high voltage TL has performance limitations due to the effect of high fault resistance at distant faults, where the pickup threshold becomes ineffective.

Distance relays usually are the priority for long TL protection compared to other protection schemes due to their reliable performance with long high voltage TLs. This scheme depends on measuring the impedance of the protected TL to identify the faulted zone. However, [2] recognized that distance relays have some obstacles regarding the phase shift between voltage and current, fault resistance and third zone maloperation [3]. The CT saturation effect appears prominently in the transmission network using distance protection schemes; hence it results in excessive tripping delay time [4-6]. The high fault current in some cases prevents CTs from sending the actual current value to the relay, and the relay will receive only a relatively low current at the secondary side during severe faults. However, distance protection is recommended for distribution networks due to it having a directional element that increases its ability to deal with meshed networks. The high cost of this scheme might be an obstacle since it requires both voltage and current transformers. However, it is only suitable for a small range of considerations and the correct settings may be more difficult to determine compared to the OCR [1].

Differential relays have also gained a wide recognition because it is a protection scheme rated as highly sensitive, selective, fast and insensitive to the bi-directional flow of current when compared to the distance and overcurrent schemes [1], [7]. The differential relay operational concept is based on calculating currents from the connected CTs across the protected section according to Kirchhoff's law. Differential relays face some issues due to the fault location discrimination besides the effect of $\mathrm{CT}$ saturation and $\mathrm{CT}$ mismatch on the relay operation [8]. Communication failures because of limited bandwidth channels over long distances also play a major role in reducing the effectiveness of this scheme. However, compared to distance relays it can be applied to different line lengths, and is more effective with medium and short length lines. Reference [9] pointed out that a differential relay is adaptable to changing system conditions and continuous development such as inverter-interfaced power/energy sources. In addition, differential relays operate efficiently with multi-terminal compensated lines.

All the previously mentioned protection schemes are current-based approaches depending on CT measurements, which adversely affects the accuracy of these schemes when the CT saturates during severe fault events. During faults, the high current will cause the current in the secondary winding of the CT to increase which will increase flux proportionally; the flux will continue to increase according to current until the CT core saturates. This causes a delayed trip for faults inside the protected zone or unwanted trips for faults outside the protected zone [10], [11].

The effect of mutual coupling of parallel TLs make the operation of protection devices complicated, particularly for distance relays; the effect of this increases with unbalanced 
inter-circuit faults affecting the calculation of the line mutual impedance, hence causing a false fault indication. The mutual impedance effect is strong, it might be as high as $70 \%$ of the zero sequence impedance of the line; while the negative and positive sequence couplings have a negligible effect on protection schemes [12].

The recent vast changes in the power system networks around the world have led to a significant change in the generation mix. The use of inverter-interfaced technologies with a high number of PEDs led together to reduce the fault current level in these networks. This problem directly affects the conventional high fault current-based protection schemes and hence the system reliability as mentioned in the latest technical report of IEEE power and energy society [13].

To overcome the aforementioned issues, a voltage-based protection scheme is proposed. Very few research studies have been done to evaluate voltage-based schemes in power system protection [14], [15]. Reference [16] suggested converting voltage signals into a DC quantity in the dq frame, which is complicated and challenging.

This paper proposes a voltage-based protective scheme that is able to detect a fault and to effectively distinguish between faults inside and outside the protected zone. The main concept of the proposed scheme is based on using voltage measurement instead of current. To guarantee the proposed scheme accuracy, the negative sequence component is precisely used. Using the negative component of voltage helps to avoid the maloperation of the proposed scheme during heavy faults. The model of a Negative Sequence Voltage (NSV) based differential relay is tested with different fault scenarios on an $11 \mathrm{kV}$, radial network simulated by MATLAB/Simulink software.

The rest of the paper is organized as follows. Section II will review the concept of the traditional differential relay and section III will explain the proposed approach of the NSV-based differential protection. Then the simulation results shown in section IV illustrate the high performance of the relay under different fault types and locations. Finally, section $\mathrm{V}$ concludes the paper.

\section{DIFFERENTIAL RELAY CONFIGURATION}

A differential protection scheme consists of two independent differential relays, which are linked by a wired/wireless communication channel as shown in Fig.1

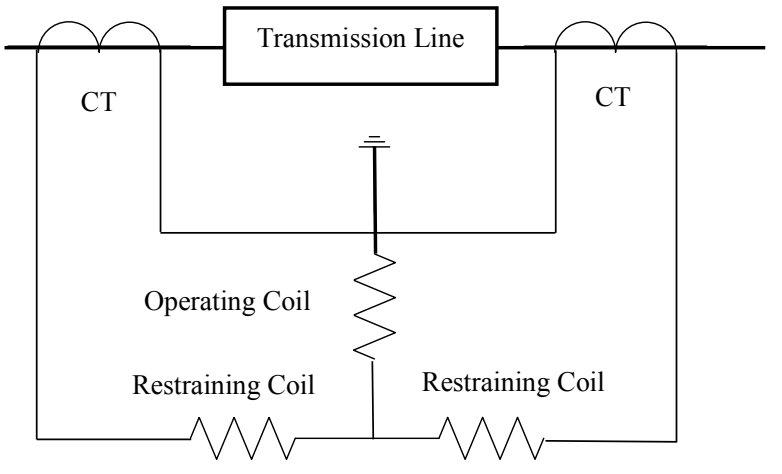

Fig. 1. Typical line differential scheme

The main principles of this protection scheme are described by (1), (2) as follows:

$$
\begin{aligned}
& \left|I_{L}+I_{R}\right|>I_{S} \\
& \left|I_{L}+I_{R}\right|>K .\left(\left|I_{L}\right|-\left|I_{R}\right| / 2\right)
\end{aligned}
$$

where $I_{L}$ and $I_{R}$ represent the current measured at the local and remote ends of the protected line, respectively. $\left|I_{L}+I_{R}\right|$ is known as operating current while $\left(\frac{\left|I_{L}-I_{R}\right|}{2}\right)$ represents the restraining current. $K$, a proportional coefficient measuring the sensitivity of the relay, is calculated as follows

$$
K=\frac{\left|I_{L}+I_{R}\right|}{\left(\left|I_{L}\right|-\left|I_{R}\right| / 2\right)}
$$

The developed technology of numerical and digital relays made the operation of the protection devices easier, using symmetrical components and data synchronization to detect faults more accurately. Reference [8] proved that negative sequence component follows Kirchhoff's law, so (1)-(3) can be readily applied to a differential relay based on negative sequence quantities. The negative sequence current from each side of the line will be calculated. Then the operating and restraining signals will be estimated as described in (1) and (2). As shown in Fig. 2 the restraint quantity will control the operation of the relay providing more stable operation for it during an external fault or under normal load condition. The fault will be detected when the operating signal exceeds the restraint quantity and the threshold current setting. When an internal fault occurs, the operating signal will exceed the restraining one. Therefore, the relay will send a trip signal to the circuit breaker to operate. The area below the Ipu in Fig. 2 represents the relay status under low operating current.

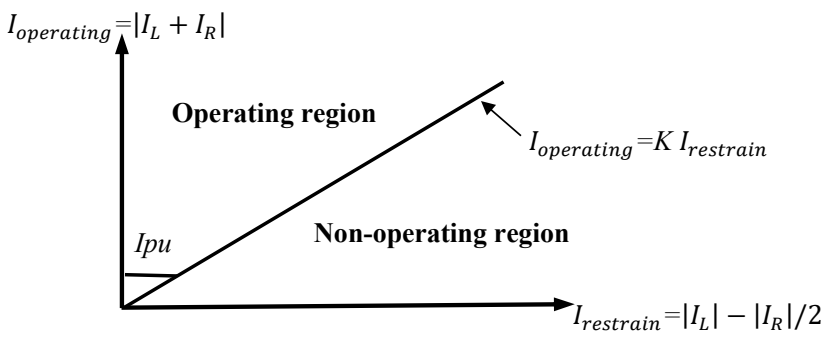

Fig. 2. Operating characteristics

\section{NSV-BASED DIFFERENTIAL RELAY}

The current-based differential relay mentioned above performs well to some extent [17-19]. However, it still suffers from issues mentioned earlier regarding the current limitation of the PEDs, and the CT saturation.

The proposed protection scheme of voltage-based differential relay basically depends on using voltage transformers (VTs) instead of CTs to avoid all the CTs issues. The proposed method is based on using Kirchhoff's voltage law (KVL). The three-phase input voltage is measured using VTs connected to the both sides of the protected section of the tested TL as shown in Fig.3. It is assumed that the voltage drop across the protected section is equal to zero during normal operation. 


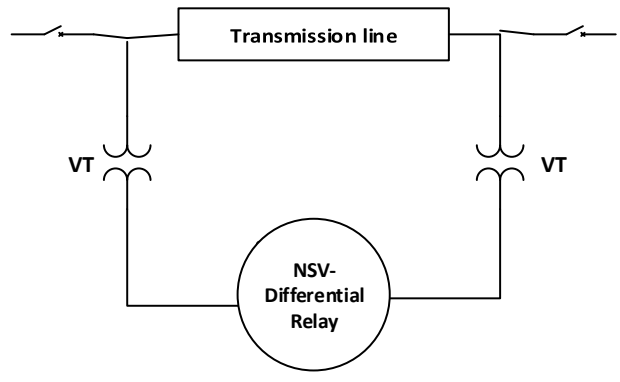

Fig. 3. NSV relay

The proposed NSV differential relay is represented in Fig.4, which relies on using NSV as the main quantity to detect a fault in the protected section of a TL.

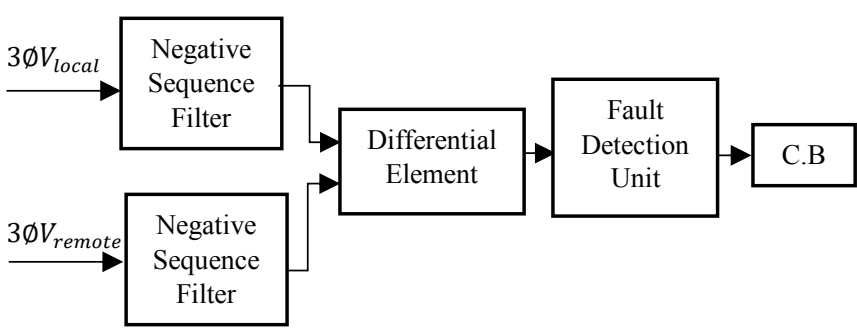

Fig. 4. NSV-based differential relay block diagram

The first step of the fault detection algorithm is to measure the NSV from both sides of the protected TL using a negative sequence filter according to (4).

$$
V^{-}=\frac{1}{3}\left(V_{a}+a^{2} V_{b}+a V_{c}\right)
$$

where $a$ represents the phase shift between the phases. Voltage difference can be calculated by applying KVL, as expressed below,

$$
V_{\text {diff }}^{-}=\left|V_{L}-V_{R}\right|
$$

where $V_{L}$ and $V_{R}$ represent the voltage at local and remote ends of the measured circuit, respectively and $V_{\text {diff }}^{-}$ represents the negative differential voltage drop.

Then set the pickup values of the NSV to guarantee the best fault location discrimination and provide enough stability for this relay operation. The measured negative voltage drops will be compared with two constants, $C_{1}$ and $C_{2}$.

$$
C_{1}<\Delta V_{\text {diff }}^{-}<C_{2}
$$

$C_{1}$ and $C_{2}$ calculation is based on many factors to evaluate the protected system in all the expected events such as the operation conditions and fault location as follows:

\section{A. Normal Operation Condition-No Fault Event}

As the first case to be considered when setting the pickup values, the maximum voltage drop for the protected system should be calculated during normal operation condition. This maximum negative voltage drop should be measured during the peak load demand where the NSV quantity will arise as the connected loads consume the highest possible current, where

$$
\Delta V_{\max -\text { load }}^{-}=Z_{\text {line }} \times I_{\text {load }}
$$

By following (8), the NSV differential relay will not trip during no-fault events.

$$
0<\Delta V_{\text {diff }}^{-}<\Delta V_{\text {max-load }}^{-}
$$

\section{B. Internal Fault Condition}

By considering the fault type and location inside the protected zone the minimum and maximum negative voltage drops are calculated. The operational voltage drop signal for an internal fault should be following (9):

$$
\Delta V_{\text {min-internal fault }}^{-}<\Delta V_{\text {diff }}^{-}<\Delta V_{\text {max-internal fault }}^{-}
$$

where $\Delta V_{\text {min-internal fault }}^{-}$is estimated during a (L-G) fault near the local VT, $(\mathrm{F} 1)$, and $\Delta V_{\max \text {-internal fault }}^{-}$is estimated during a (L-L) fault near the remote VT, (F2), as shown in Fig.5. These cases represent the worst cases to be considered when setting the pickup constants. The $\Delta V_{\text {min-internal fault }}^{-}$ should also follow (10) to endorse that the proposed scheme will not trip for a no-fault event.

$$
\Delta V_{\text {min-internal fault }}^{-}>\Delta V_{\text {max-load }}^{-}
$$

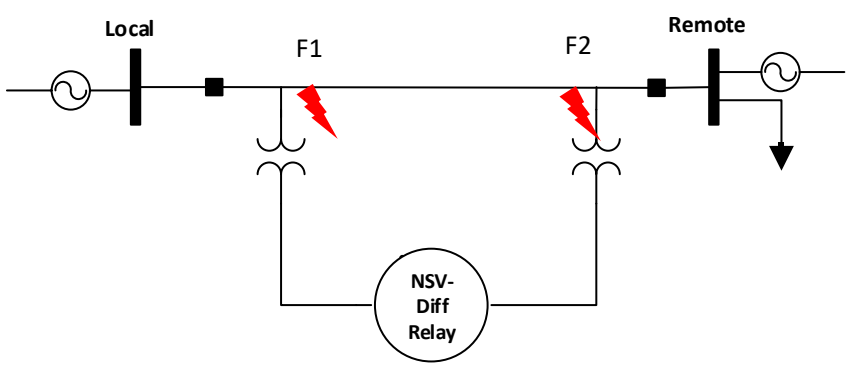

Fig. 5. NSV-based differential relay during internal fault

\section{External Fault Condition}

To ensure the reliability of the proposed scheme and finalize the calculation of pickup setting values, external faults should also be considered. The NSV drop must be measured during different external faults at the local and remote ends together. The minimum voltage drop at both sides measured during (L-G) fault, and the maximum voltage drop at both sides measured during (L-L) fault is shown in Fig.6.

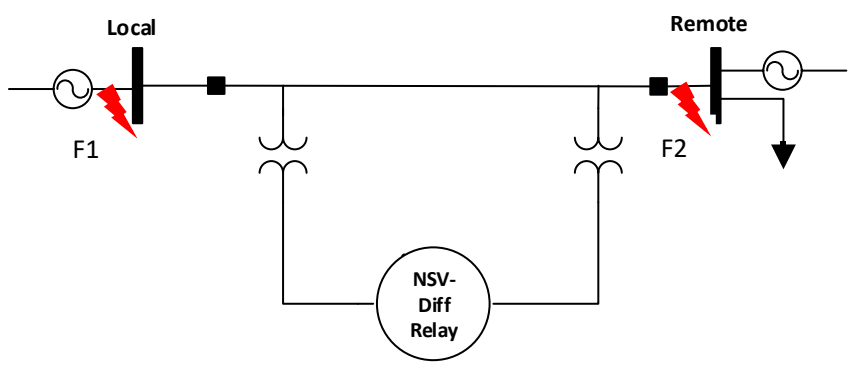

Fig. 6. NSV-based differential relay during external fault

where the measured, $\Delta V_{\text {min-external fault-remote }}^{-}$and

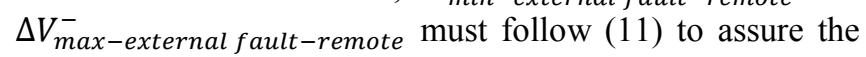
selectivity of the relay. 
where

$\left.\begin{array}{rl}\Delta V_{\text {min-external fault-remote }}^{-} & >\Delta V_{\text {max-internal fault }}^{-} \\ \text {and } & \\ \Delta V_{\text {max-external fault-remote }}^{-} & >\Delta V_{\text {max-internal fault }}^{-}\end{array}\right\}$

These restriction conditions in (8-11) together will restrain the NSV differential relay from tripping during any outside zone disturbance and any fault type. The pickup constant values then will be chosen, where $C_{1}$ must be higher than the $\Delta V_{\text {min-internal fault }}^{-}$and $C_{2}$ less than the $\Delta V_{\text {max-internal fault }}^{-}$.

The flowchart shown in Fig. 7 summaries the proposed NSV-based differential relay scheme.

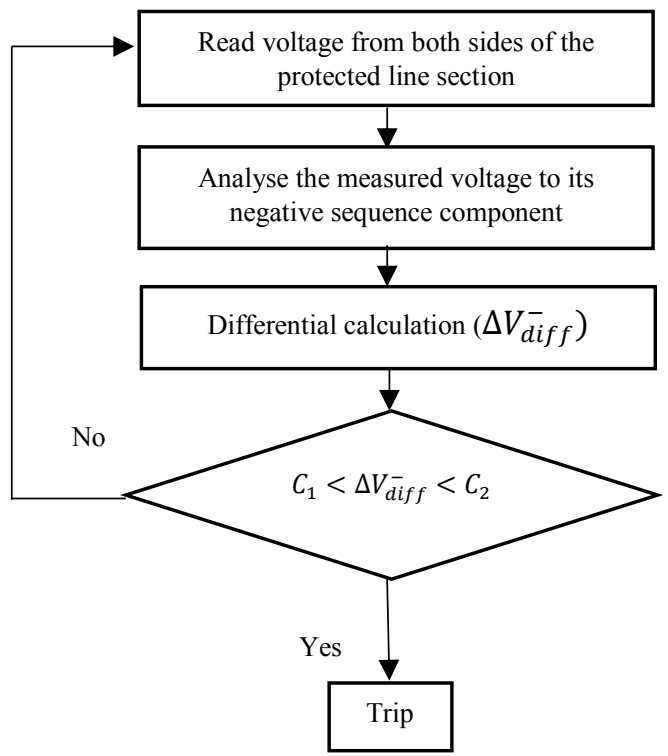

Fig. 7. Flowchart of the proposed scheme

\section{SIMULATION RESULTS}

As shown in Fig. 8, the model including $11 \mathrm{kV}$ generator, a load of $10 \mathrm{MW}$ and 100 MVAR at the remote end, and a transmission line section of $60 \mathrm{~km}$ long is simulated by MATLAB. The frequency of the tested system is $50 \mathrm{~Hz}$.

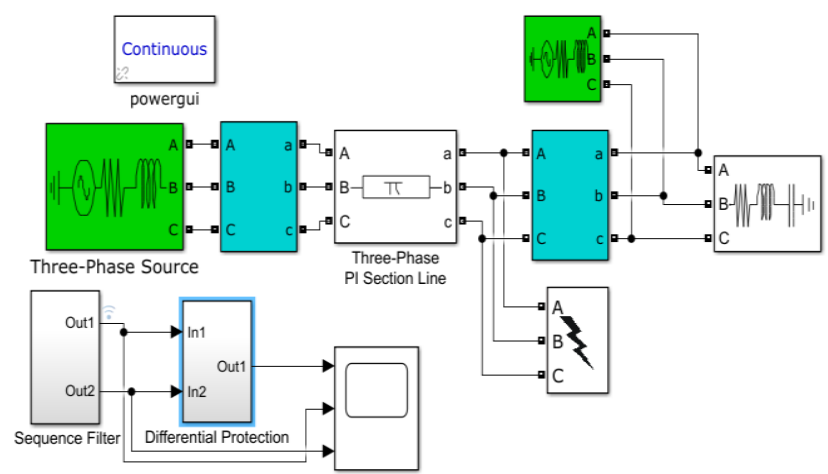

Fig. 8. The Simulink model of the tested system

\section{Scenario1. No-Fault Condition}

Fig. 9 shows that the negative voltage measurements from local and remote ends are very small or negligible. The NSV differential relay does not send a trip signal so that the tripping signal is also zero as illustrated in Fig.9. After several simulations, a very small amount of the negative voltage drop is measured when the system is fully loaded, i.e. $\Delta V_{\max -\text { load }}^{-}$ is measured as $4.16075 \mu \mathrm{V}$ as shown in Fig. 10 .

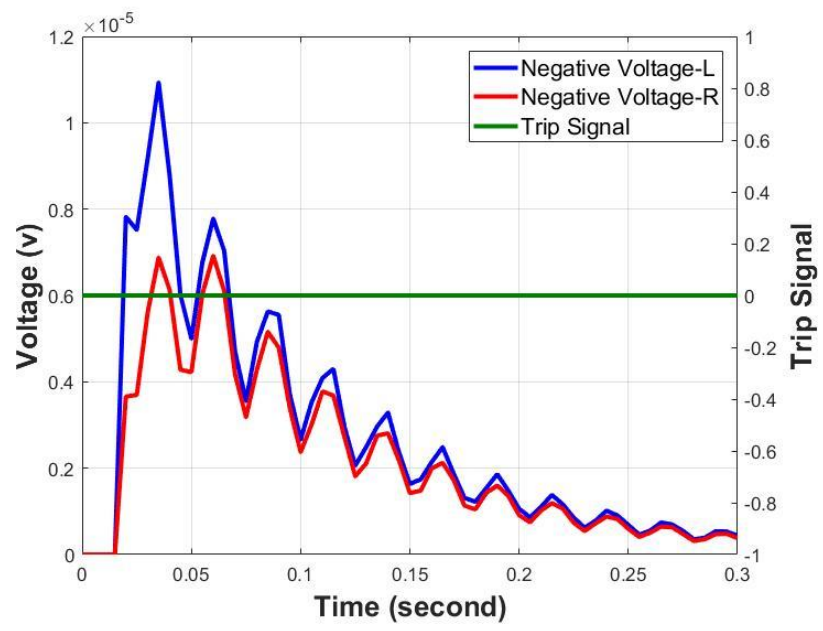

Fig. 9. Simulation results when no fault occurs

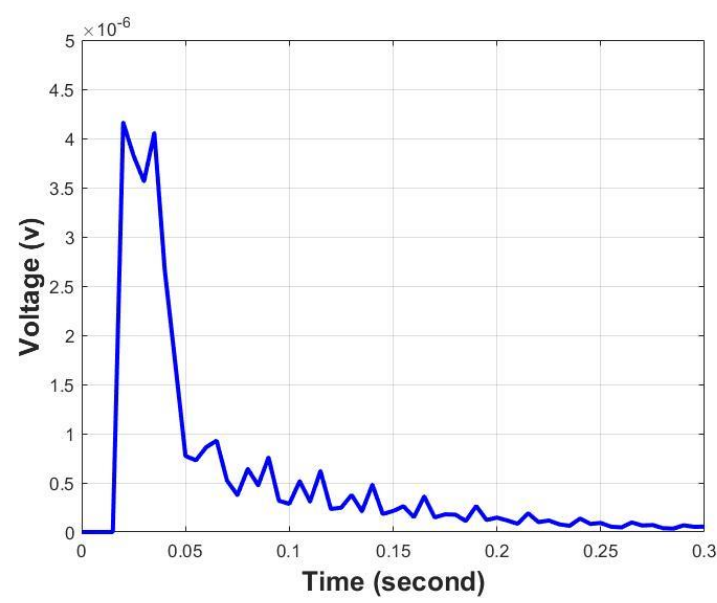

Fig. 10. Maximum negative voltage drop during peak load

\section{Scenario2. Internal Faults}

Fig. 11, a, b and c show the simulation results for three types of asymmetrical faults, i.e. line-line, line-ground and line-line-ground, applied to the TL and illustrates how the negative drop voltage detect them accurately when a fault happens at time $t=0.05 \mathrm{sec}$.

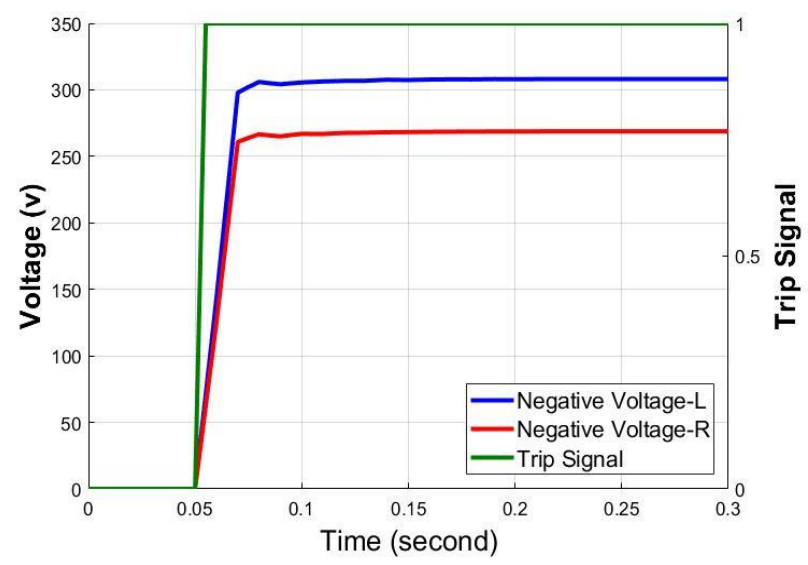

(a) Internal (L-G) fault 


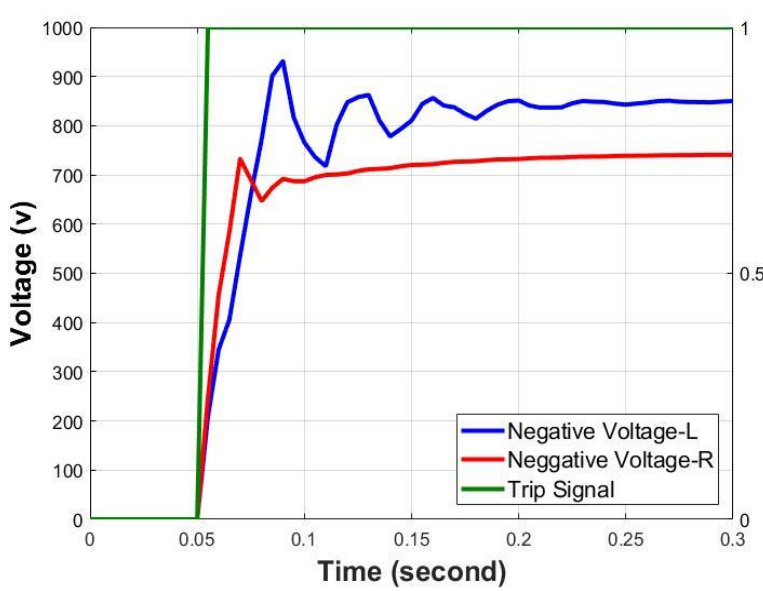

(b) Internal (L-L-G) fault

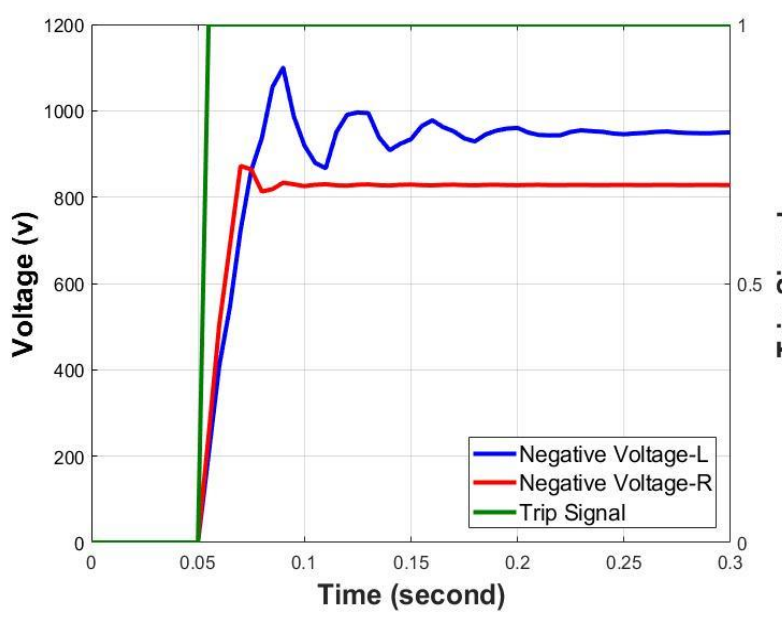

(c) Internal (L-L) fault

Fig. 11. Simulation results when internal fault occur

\section{Scenario3. External Fault}

Fig. 12 shows that when an external fault occurs at $t=$ $0.05 \mathrm{sec}$ there will be clearly a high negative voltage drop in the system, but the relay will not trip since the drop voltage is out of the restriction conditions of the designed relay, where either the $\Delta V_{\text {min-external fault-remote }}^{-}$is less than $C_{1}$ or the $\Delta V_{\text {max-external fault-remote }}^{-}$is higher than $C_{2}$

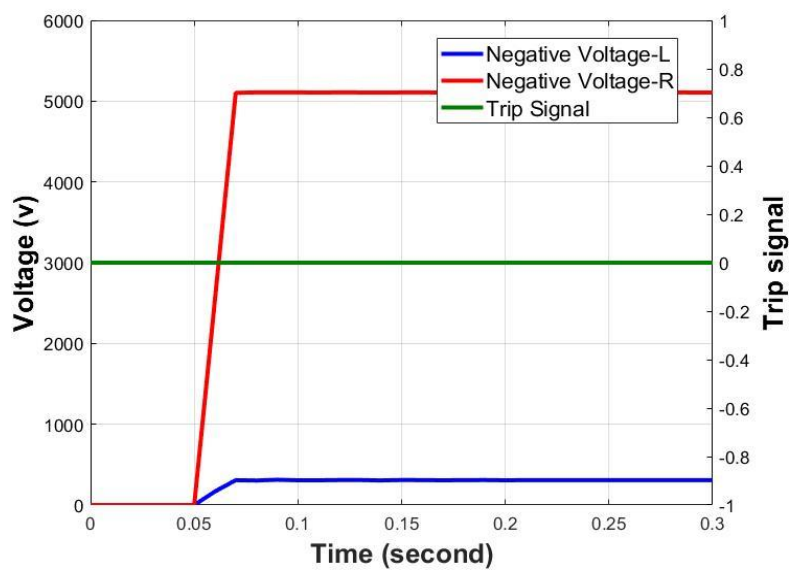

(a) External (L-G) fault at the remote side

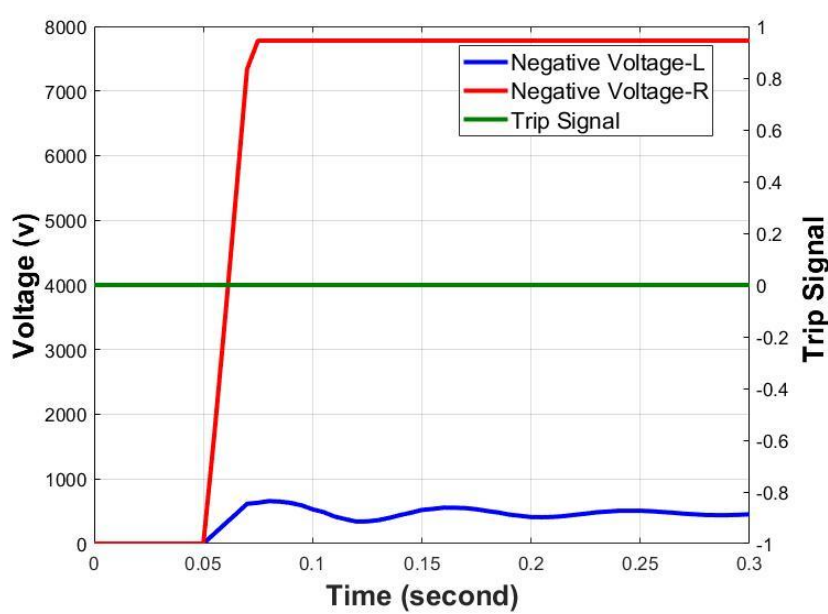

(b) External (L-L) fault at the remote side

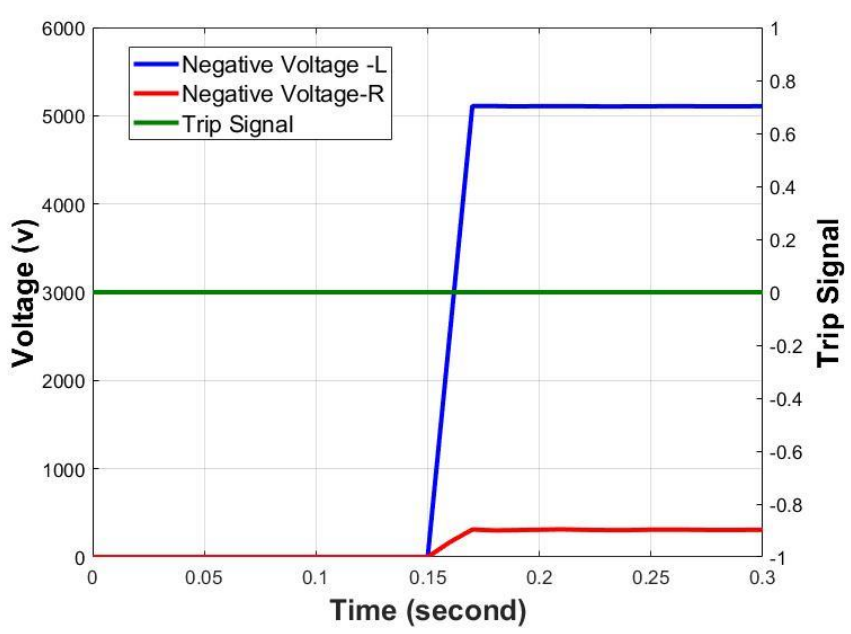

(c) External (L-G) fault at the local side

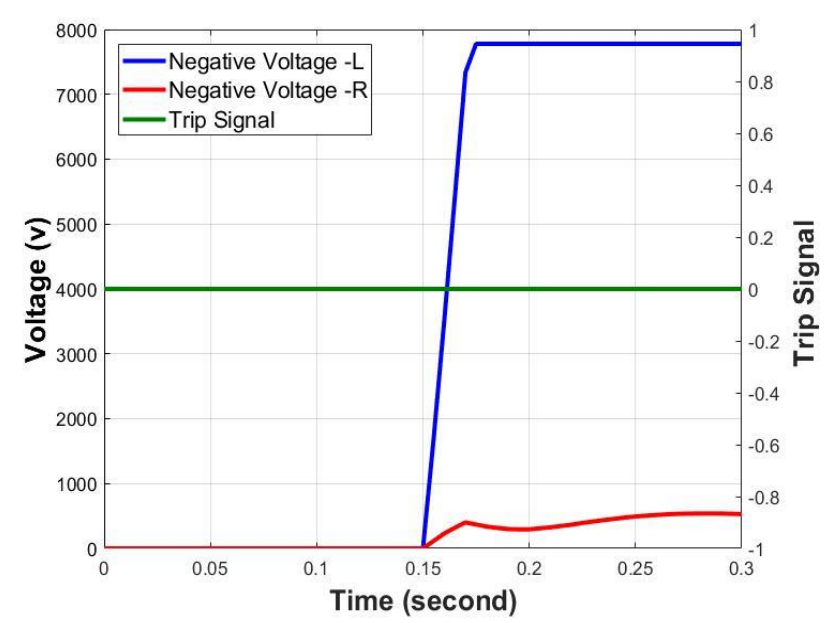

(d) External (L-L) fault at the local side

Fig. 12. Simulation results when external fault occur

Considering the worst fault cases, the pickup values are determined to be $C_{1}=0.5$ and $C_{2}=1000$. The three scenarios considered showed the selectivity of the proposed scheme, where the relay did not trip for any abnormal event except for faults inside the section bounded by the VTs. 
The conventional current-based differential relay operates perfectly to some extent compared to other protection schemes and works even better when considering sequence components as described in [7], [20], [21]. However, the tendency of differential relays to fail in the real world during heavy fault events is high. In addition, the continued penetration of inverter-based resources makes it necessary to start looking for alternative schemes. Suggested protection methods must be less depending on current due to the upcoming challenges related to reduced level of fault current. The reduced fault current might cause a serious system stability problem. The proposed method also can be used in the high voltage part of the power network where negative voltage gives a better indication; and where the negative sequence current and current generally have many restrictions on this side of the power system network.

\section{CONCLUSION}

This paper describes a model of NSV-based differential relay and its working concepts as a differential protection scheme for TL systems for medium/low voltage levels. The proposed relay was tested for different fault types and locations and it showed high discrimination accuracy between internal and external faults. The main challenge of the proposed method is calculating and determining suitable settings to make the proposed NSV-based differential relay operate properly. Improving the model operation and accuracy is recommended for future research.

\section{ACKNOWLEDGMENT}

The authors acknowledge funding support to carry out this research from University of Auckland Doctoral Scholarship; University of Auckland under Faculty Research Development Fund (FRDF) Grant 370953; and, Ministry of Business, Innovation and Employment, New Zealand's National Science Challenge-Resilience to Nature's Challenge and in particular the project on Electricity Distribution Resilience Framework informed by West Coast Alpine Fault Scenario.

\section{REFERENCE}

[1] M. Norshahrani, H. Mokhlis, A. Abu Bakar, J. Jamian, and S. Sukumar, "Progress on protection strategies to mitigate the impact of renewable distributed generation on distribution systems," Energies, vol. 10, no. 1864, 2017.

[2] H. Herrmann, "Impact by more inverter based power penetration on protection and control," CIGRE study committee B5 meeting and colloquium, NewZealand, 2017.

[3] A.R.Motavalian, N.Moadabi, and G. B. Gharehpetian, "Reliability assessment of power system backup protection in smart grid control center using phasor measurement units (PMU)," International Conference on Renewable Energies and Power Quality, Spain vol. 1, no. 11, pp.404-410, 2013.

[4] P. Jena, A. K. Pradhan, "Reducing current transformer saturation effect in phasor measurement unit," International Transactions on Electrical Energy Systems, vol. 26, no. 7, pp. 1397-1407, 2015.

[5] K. Wannous and P. Toman, "The impact of current transformer saturation on the distance protection," 17th International Scientific Conference on Electric Power Engineering, pp. 1-5, 2016.

[6] J. Mooney, "Distance element performance under conditions of CT saturation," 61st Annual Conference for Protective Relay Engineers, pp. 491-497, 2008.

[7] H. Miller, J. Burger, N. Fischer, and B. Kasztenny, "Modern line current differential protection solutions," 63rd Annual Conference for Protective Relay Engineers, pp.1-25, 2010.
[8] N. Zhang, X. Dong, Z. Bo, and A. Klimek, "Performance comparison of current differential protection scheme based on symmetrical components," 42nd International Universities Power Engineering Conference, UK, pp. 305-309, 2007.

[9] J. Roberts, D. Tziouvaras, G. Benmouyal, and H. Altuve, "The effect of multiprinciple line protection on dependability and security," 54th Annual Conference for Protection Relay Engineers, 2001.

[10] S. Holst, I. Brnčič, D. Shearer, R. Mangelred, K. Koreman, "Problems and solutions for ac transmission line protection under extreme conditions caused by very long HVDC cables," CIGRE study committee B5 meeting and colloquium, Spain, pp. 1-8, 2007.

[11] A. Hargrave, M. J. Thompson, and B. Heilman, "Beyond the knee point : a practical guide to CT saturation," 71st Annual Conference for Protective Relay Engineers, pp. 1-23, 2018.

[12] V. H. Makwana, and B. R. Bhalja, "A new adaptive distance relaying scheme for mutually coupled series-compensated parallel transmission lines during intercircuit faults," IEEE Transactions on Power Delivery, vol. 26 , no. 4,2011 .

[13] IEEE PES-TR68, "Impact of inverter based generation on bulk power system dynamics and short-circuit performance," July, 2018.

[14] K. Haddad, G. Joos, and S. Chen, "Control algorithms for series static voltage regulators in faulted distribution systems," 30th Annual IEEE Power Electronics Specialists Conference. Record. (Cat. No.99CH36321), vol.1, pp. 418-423, 1999.

[15] P. T. Nguyen, and T. K. Saha, "Dynamic voltage restorer against balanced and unbalanced voltage sags: modelling and simulation," IEEE Power Engineering Society General Meeting, vol. 1, pp. 639644, 2004.

[16] H. AL-Nasseri, and M. A. Redfern, "A new voltage based relay scheme to protect micro-grids dominated by embedded generation using solid state converters.," 19th International Conference on Electricity Distribution, no. 723, pp. 21-24, 2007.

[17] D. Bejmert, W. Rebizant, and A. Wiszniewski, "Enhanced differential protection algorithm for tapped transmission lines," IEEE Trondheim PowerTech., pp.1-8, 2011.

[18] Y. Xue, B. Kasztenny, D. Taylor and Y. Xia, "Line differential protection under unusual system conditions," Line Current Differential Protection: A Collection of Technical Papers Representing Modern Solutions, 2014, Section II, pp. 1-17, 2014.

[19] H. Seyedi and S. AsghariGovar, "Adaptive CWT-based transmission line differential protection scheme considering cross-country faults and CT saturation," IET Generation, Transmission \& Distribution, vol. 10, no. 9, pp. 2035-2041, 2016.

[20] I. A. Rizvi and G. Reeser, "Using symmetrical components for internal external fault discrimination in differential protection schemes," 66th Annual Conference for Protective Relay Engineers, pp. 68-79, 2013.

[21] B. Kasztenny, N. Fischer, and H. J. Altuve, "Negative-sequence differential protection-principles, sensitivity, and security," 68th Annual Conference for Protective Relay Engineers, no. 1, pp. 364-378, 2015 . 